\title{
Marine-Derived Fungi: A Promising Source of Halo Tolerant Biological Control Agents against Plant Pathogenic Fungi
}

\author{
Tanaporn Chalearmsrimuang ${ }^{1}$, Siti Izera Ismail ${ }^{2}$, Norida Mazlan ${ }^{3}$, Supaporn \\ Suasaard ${ }^{1}$ and Tida Dethoup ${ }^{1 *}$ \\ ${ }^{1}$ Department of Plant Pathology, Faculty of Agriculture, Kasetsart University, Bangkok 10900, Thailand. ${ }^{2}$ Department \\ of Plant Protection, Faculty of Agriculture, Universiti Putra Malaysia, 43400 Serdang, Malaysia. ${ }^{3}$ Department of \\ Agriculture Technology, Faculty of Agriculture, Universiti Putra Malaysia, 43400 Serdang, Malaysia.
}

\begin{abstract}
In this study, twenty marine-derived fungi were evaluated for their antagonistic activities against 10 economically important plant pathogenic fungi and investigated for their halo tolerance on potato dextrose agar (PDA) amended with $1 \%-25 \% \mathrm{NaCl}$. The results of dual culture tests showed that the marine Trichoderma species, $T$. asperellum and $T$. harzianum exhibited higher antagonistic effects against all plant pathogens than the other tested fungi, causing percentages of mycelial growth inhibition ranging from $59.31-100 \%$. The results of dilution plate assays revealed that crude extracts of marine-derived fungi in the genera Emericella, Myrothecium, Neocosmospora, Penicillium and Talaromyces displayed great antifungal activity against plant pathogenic fungi at a low concentration of $1 \mathrm{~g} / \mathrm{L}$. However, the crude extract of Myrothecium verrucaria showed the best antifungal activity: more than $\mathbf{5 2 \%}$ inhibition of five of the tested species of plant pathogenic fungi and complete mycelial growth inhibition of Bipolaris oryzae and Lasiodiplodia theobromae at $1 \mathrm{~g} / \mathrm{L}$. All of the tested marinederived fungi were tolerant to $\mathrm{NaCl}$ at concentrations up to $7 \%$. These results revealed marine-derived fungi possess exploitable antagonistic activities against plant pathogenic fungi through antibiosis, competition for nutrients and space and halo tolerance. Moreover, the results from this study showed their potential as novel BCAs for supporting crop production under climatic changes in the future.
\end{abstract}

Keywords: Antagonistic activities; marine fungi; plant pathogens; halo tolerant fungi.

*Correspondence: agrtdd@ku.ac.th; +66 25761026, +66 924379991.

(Received: 07 January 2019; accepted: 07 February 2019)

Citation: Tanaporn Chalearmsrimuang, Siti Izera Ismail, Norida Mazlan, Supaporn Suasa-ard and Tida Dethoup, Marine-Derived Fungi: A Promising Source of Halo Tolerant Biological Control Agents Against Plant Pathogenic Fungi, J Pure Appl Microbiol., 2019; 13(1):209-223 doi: 10.22207/JPAM.13.1.22

C The Author(s) 2019. Open Access. This article is distributed under the terms of the Creative Commons Attribution 4.0 International License which permits unrestricted use, sharing, distribution, and reproduction in any medium, provided you give appropriate credit to the original author(s) and the source, provide a link to the Creative Commons license, and indicate if changes were made. 


\section{INTRODUCTION}

The disadvantages of commercial synthetic fungicides in both organic and conventional farming have led to attempts to find new strategies for controlling plant diseases ${ }^{1,2}$. Biological control agents are currently held to be a very promising strategy for plant disease management due to their being eco-friendly and non-toxic to consumers and farmers ${ }^{3,4}$. Finding novel BCAs is required to combat plant disease outbreaks and over come plant pathogen resistance to fungicides. The search for promising BCAs has mostly been conducted by screening terrestrial, endophytic, entomopathogenic microbes while studies of antagonistic microbes from marine environments are still limited. Marine invertebrates present a rich source of bioactive metabolites ${ }^{5,6}$. Moreover, they are also the major hosts of symbiotic microorganisms such as actinomyces, bacteria and fungi ${ }^{7,8}$. Marine-derived fungi are often associated with marine organisms and substrata such as sponges, corals, tunicates, higher algae, sea grasses, mangroves, molluscs, woody substrates and drift wood ${ }^{9,10}$.

In our ongoing search for bioactive compounds from marine-derived fungi, we isolated a number of fungi from sponges, corals and sea fans, among which was a novel fungal species recently reported ${ }^{11}$. Several novel metabolites and the antimicrobial activity of marine-derived fungi isolated from marine invertebrates collected from Thai waters against human and plant pathogens have been reported by our group ${ }^{12-15}$. Fungi isolated from marine environments, particularly from sponges, have shown great potential as important sources of pharmacologically active metabolites and biological activities which have great potential for the development of new drugs as well as new agrochemical substances ${ }^{16-18}$. They have also been reported to be more important producers of novel natural products and bioactive compounds than other microorganisms ${ }^{19-23}$.

These new bioactive compounds are attracting researchers to attempt to isolate fungi from marine environments. These fungi have previously been isolated from soils and plants in different locations and climates. To date, studies of diversity in marine organisms have led to the isolation of hundreds of fungal species belonging to Ascomycetes, Deuteromycetes, Zygomycetes and Mitosporic fungi ${ }^{24-28}$. Most of them were previously reported as terrestrial fungi, and they were able to grow on media both with and without the addition seawater ${ }^{11,16}$. The fungi and the marine invertebrate, plant relationship is still unclear; however, sponge derived fungi have classified into three groups: sponge-generalists, sponge-associates and sponge-specialists ${ }^{27,29}$.

In our previous study, we reported the in vitro antifungal activity of five marinederived fungi against 10 economically important plant pathogens. Among these, the extract of Talaromyces trachysporus isolated from the marine sponge Clathria reinwardtii had great mycelial growth inhibition capability on Pythium aphanidermatum even at the low concentration of $\mathrm{IC}_{50} 100 \mathrm{ppm}^{10}$. Besides this, other researchers have investigated the antibacterial and antifungal properties of marine-derived fungi against plant pathogens $^{30-32}$. For example, several Trichoderma spp. were isolated from the Mediterranean sponge, Psammocinia sp., and were evaluated for their antagonistic activity against three plant pathogenic fungi, Botrytis cinerea, Rhizoctonia solani and Alternaria alternata. The results showed that all the tested fungi extracts displayed antagonistic activity in dual plate assays. T. atroviride and $T$. asperelloides effectively reduced the incidence of $R$. solani damping-off disease of beans and also induced defense responses in cucumber seedlings against Pseudomonas syringae pv. lachrimans ${ }^{33}$.

These data showed that marine-derived fungi, and especially marine sponge-associated fungi, are a promising source of antagonist microbes which may be useful in developing as novel BCAs to control plant diseases. However, their antimicrobial properties were mostly demonstrated for pharmaceutical purposes; thus, the evaluation of antagonistic activity against plant pathogens in this study may provide more information concerning the value and potential of marine-derived fungi in crop protection. The purpose of this study was to evaluate the antagonistic activities and halo tolerance of twenty selected marine-derived fungi collected from Thai waters against ten plant pathogenic fungi in vitro. 


\section{MATERIALS AND METHODS Sponge samples}

The marine sponge samples were collected from coral reefs at two locations in Thailand: Samaesan Island, Chonburi Province in Eastern Thailand and Similan Island, Phang Nga Province, in Southern Thailand, by scuba- diving at a depth of 10-15meters during 2011-2016 (Table 1). The samples were placed in plastic bags containing natural seawater and were stored in ice and in a refrigerator for later analysis.

\section{Isolation of fungi from marine sponges}

The sponge sample tissues were washed three times with sterilized sea water and cut into pieces of $0.5 \times 0.5 \mathrm{~cm}$ under aseptic conditions. Five pieces of each marine sponge were placed on a Petri dish plate containing $15 \mathrm{~mL}$ malt extract agar (MEA) medium mixed with70\% sea water and $0.003 \%$ streptomycin sulphate, and then incubated at room temperature for 7 days. Hyphal tips emerging from sponge pieces were cut and transferred to MEA slants for further identification. The pure cultures were maintained at the Department of Plant Pathology, Faculty of Agriculture, Kasetsart University, Bangkok, Thailand under the code KUFA.

\section{Marine-derived fungi identification}

The identification of the fungi was based on morphological characteristics as observed from the growth pattern, color and texture on MEA. Colony characteristics were examined under a stereoscopic microscope, and microscopic characteristics were thoroughly investigated under light and scanning electron microscopes afterwards. The fungi were further identified by molecular techniques using ITS primers. DNA was extracted from young mycelia following a modified Murray and Thompson method ${ }^{34}$.Universal primer pairs ITS1 and ITS4 were used for ITS gene amplification ${ }^{35}$. The gene sequences of the marine-derived fungi were submitted to the BLAST program for alignment and compared with those of fungal species in the NCBI database (http://www.ncbi.nlm.nih.gov/). Their ITS gene sequences were deposited in GenBank with accession numbers as shown in Table 1.

In vitro antagonistic activity testing of the marinederived fungi against plant pathogenic fungi by the dual culture method

Twenty marine-derived fungi were selected for testing of their antagonistic activity against ten species of plant pathogenic fungi (Table 2). The marine-derived fungi and plant pathogenic fungi were cultured on separate Petri dish plates containing PDA and incubated at room temperature for 7 days. A mycelial plug of marine-derived fungus and a mycelial plug of plant pathogenic fungus were cut from the colony margin with a sterile steel borer $(0.5 \mathrm{~cm}$ diam.) and placed on PDA as a dual culture, $7 \mathrm{~cm}$ apart. The Petri dish plates of the dual culture assay were incubated at room temperature for 3 days for Sclerotium rolfsii and Rhizoctonia solani, and for 14 days for the other species. A mycelial plug of each plant pathogenic fungus was placed on a separate PDA plate to serve as a control. The inhibition levels were calculated by using the formula: [ $x$ $y) / x] \times 100$, where $x=$ the colony radius of the plant pathogenic fungi in the control, and $y=$ the colony radius of the plant pathogenic fungi in the dual culture test. Each treatment was performed with five replicates and repeated three times.

Preparation of the marine-derived fungal extracts The 20 selected marine-derived fungi were evaluated for their antifungal activity against plant pathogenic fungi (Table 3). These fungi were cultured on separate PDA plates and incubated at room temperature for 7 days. Five mycelial plugs of each fungus were cut from a 7-day-old colony margin and inoculated in $500 \mathrm{ml}$ Erlenmeyer flasks containing potato dextrose broth $200 \mathrm{~mL}$, and then incubated on a rotary shaker at $120 \mathrm{rpm}$ for 7 days for preparing spore suspensions. Twenty-five $1,000 \mathrm{ml}$ Erlenmeyer flasks, each containing $300 \mathrm{~g}$ cooked rice, were autoclaved at $121^{\circ} \mathrm{C}$ for $15 \mathrm{~min}$. and then inoculated with approximately $20 \mathrm{~mL}$ of mycelial suspension of each fungus. The inoculated flasks were then incubated at room temperature for 30 days, after which $500 \mathrm{~mL}$ of ethyl acetate was added to each flask and macerated for 7 days. The ethyl acetate solutions were filtered through filter paper (Whatman No.1) to give the organic solutions and then evaporated under reduced pressure to obtain a crude ethyl acetate extract of each marine-derived fungus.

In vitro antifungal activity test of marine-derived fungi crude extracts against plant pathogenic fungi

The dilution plate method was used for the evaluation of the in vitro antifungal activity 
Table 1. Fungi isolated from marine invertebrates used in this study

\begin{tabular}{|c|c|c|c|c|}
\hline $\begin{array}{l}\text { Marine- } \\
\text { derived } \\
\text { fungus }\end{array}$ & KUFA & $\begin{array}{l}\text { Accession } \\
\text { No. }\end{array}$ & Sponge & Location \\
\hline $\begin{array}{l}\text { Arthrinium } \\
\text { xenocordella }\end{array}$ & 1018 & KY041870 & $\begin{array}{l}\text { Unidentified } \\
\text { marine sponge } \\
\text { No. } 1\end{array}$ & $\begin{array}{l}\text { Samaesan Island, } \\
\text { Chonburi }\end{array}$ \\
\hline $\begin{array}{l}\text { Eurotium } \\
\text { chevalieri }\end{array}$ & 0464 & KY942148 & Rhabdermia sp. & $\begin{array}{l}\text { Similan Island, } \\
\text { Phang Nga }\end{array}$ \\
\hline $\begin{array}{l}\text { Emericella } \\
\text { foveolata }\end{array}$ & 1003 & KY041869 & $\begin{array}{l}\text { Xestospongia } \\
\text { testudinaria }\end{array}$ & $\begin{array}{l}\text { Samaesan Island, } \\
\text { Chonburi }\end{array}$ \\
\hline $\begin{array}{l}\text { Emericella } \\
\text { nidulans }\end{array}$ & 0031 & MF614160 & Mycale armata & $\begin{array}{l}\text { Samaesan Island, } \\
\text { Chonburi }\end{array}$ \\
\hline $\begin{array}{l}\text { Emericella } \\
\text { rugulosa }\end{array}$ & 1002 & KY041871 & Acanthella sp. & $\begin{array}{l}\text { Samaesan Island, } \\
\text { Chonburi }\end{array}$ \\
\hline $\begin{array}{l}\text { Emericella } \\
\text { variecolor }\end{array}$ & 0261 & MF614163 & $\begin{array}{l}\text { Xestospongia } \\
\text { testudinaria }\end{array}$ & $\begin{array}{l}\text { Samaesan Island, } \\
\text { Chonburi }\end{array}$ \\
\hline $\begin{array}{l}\text { Hamigera } \\
\text { avellanea }\end{array}$ & 0450 & KY942147 & Acanthella sp. & $\begin{array}{l}\text { Samaesan Island, } \\
\text { Chonburi }\end{array}$ \\
\hline $\begin{array}{l}\text { Hamigera } \\
\text { terricola }\end{array}$ & 0214 & KU500029 & $\begin{array}{l}\text { Xestospongia } \\
\text { testudinaria }\end{array}$ & $\begin{array}{l}\text { Samaesan Island, } \\
\text { Chonburi }\end{array}$ \\
\hline $\begin{array}{l}\text { Geosmithia } \\
\text { lavendula }\end{array}$ & 0319 & KY942145 & $\begin{array}{l}\text { Stylissa } \\
\text { flabelliformis }\end{array}$ & $\begin{array}{l}\text { Samaesan Island, } \\
\text { Chonburi }\end{array}$ \\
\hline $\begin{array}{l}\text { Myrothecium } \\
\text { verrucaria }\end{array}$ & 0192 & KY942146 & Mycale sp. & $\begin{array}{l}\text { Samaesan Island, } \\
\text { Chonburi }\end{array}$ \\
\hline $\begin{array}{l}\text { Neocosmospora } \\
\text { vasinfecta var. } \\
\text { vasinfecta }\end{array}$ & 1004 & KY041868 & Mycale sp. & $\begin{array}{l}\text { Samaesan Island, } \\
\text { Chonburi }\end{array}$ \\
\hline $\begin{array}{l}\text { Penicillium } \\
\text { aculeatum }\end{array}$ & 0201 & MF614161 & $\begin{array}{l}\text { Xestospongia } \\
\text { testudinaria }\end{array}$ & $\begin{array}{l}\text { Samaesan Island, } \\
\text { Chonburi }\end{array}$ \\
\hline $\begin{array}{l}\text { Neosartorya } \\
\text { fischeri }\end{array}$ & 0107 & KY942143 & Rhabdermia sp. & $\begin{array}{l}\text { Similan Island, } \\
\text { Phang Nga }\end{array}$ \\
\hline $\begin{array}{l}\text { Neosartorya } \\
\text { pseudofischeri }\end{array}$ & 0061 & KY942144 & Hyrtios erecta & $\begin{array}{l}\text { Similan Island, } \\
\text { Phang Nga }\end{array}$ \\
\hline $\begin{array}{l}\text { Neosartorya } \\
\text { quadricincta }\end{array}$ & 0081 & KT201525 & $\begin{array}{l}\text { Xestospongia } \\
\text { testudinaria }\end{array}$ & $\begin{array}{l}\text { Samaesan Island, } \\
\text { Chonburi }\end{array}$ \\
\hline $\begin{array}{l}\text { Neosartorya } \\
\text { tsunodae }\end{array}$ & 0052 & KT201524 & Aka coralliphaga & $\begin{array}{l}\text { Similan Island, } \\
\text { Phang Nga }\end{array}$ \\
\hline $\begin{array}{l}\text { Talaromyces } \\
\text { tratensis }\end{array}$ & 0091 & KT728350 & Mycale sp. & $\begin{array}{l}\text { Samaesan Island, } \\
\text { Chonburi }\end{array}$ \\
\hline $\begin{array}{l}\text { Talaromyces } \\
\text { stipitatus }\end{array}$ & 0207 & KU500028 & $\begin{array}{l}\text { Stylissa } \\
\text { flabelliformis }\end{array}$ & $\begin{array}{l}\text { Samaesan Island, } \\
\text { Chonburi }\end{array}$ \\
\hline $\begin{array}{l}\text { Trichoderma- } \\
\text { asperellum }\end{array}$ & 0677 & KY942142 & Mycale sp. & $\begin{array}{l}\text { Samaesan Island, } \\
\text { Chonburi }\end{array}$ \\
\hline $\begin{array}{l}\text { Trichoderm- } \\
\text { aharzianum }\end{array}$ & 0689 & MF614160 & Hyrtios erecta & $\begin{array}{l}\text { Similan Island, } \\
\text { Phang Nga }\end{array}$ \\
\hline
\end{tabular}

against ten plant pathogenic fungi. One gram of each of the crude ethyl acetate extracts of marinederived fungi was dissolved in $1 \mathrm{~mL}$ dimethyl sulfoxide and serially diluted with sterile distilled water to prepare stock solutions of 100 and $10 \mathrm{~g} / \mathrm{L}$ concentrations. One $\mathrm{mL}$ of each stock solution was added to $9 \mathrm{~mL}$ of warm PDA, mixed, and poured into the Petri dishes to obtain final concentrations of 10 and $1 \mathrm{~g} / \mathrm{L}$. A mycelial plug of each of the ten plant pathogenic fungi was cut from a 7-dayold colony margin with a sterile steel borer and transferred to a PDA plate containing one of the 
concentrations of each crude extract. All the Petri dishes were incubated at room temperature for 14 days. A PDA plate void of the fungal crude extract was used as a control. The inhibition levels were calculated using the formula: $[(x-y) / x] \times 100$, where $x=$ the colony radius of the plant pathogenic fungi in the control, and $y=$ the colony radius of the plant pathogenic fungi in the presence of the tested crude extract. Each treatment was performed with five replications and repeated three times.

\section{Salt tolerance assay}

The selected twenty marine-derived fungi were evaluated for their halo tolerance on PDA amended with $\mathrm{NaCl}$ (Sigma-Aldrich ${ }^{\circ}$ ) concentrations at $1 \%, 3 \%, 5 \%, 7 \%, 9 \%, 12 \%$, $15 \%, 17 \%, 20 \%$ and $25 \%$. A mycelial plug of each marine-derived fungus was placed on the center of a PDA plate containing each $\mathrm{NaCl}$ concentration and incubated for 30 days at room temperature. The mycelial growth of each marine-derived fungus was observed and recorded at 21 days as compared with the control $(0 \%)$. Each treatment was performed with five replications and repeated three times.

\section{Statistical analysis}

Due to the non-significant differences between the repeated experiments of each treatment at $p<0.05$, data obtained from the repeated experiments were pooled and submitted to analysis of variance (ANOVA), and means were compared by Duncan's multiple range test ( $p$ $<0.05)$, using the statistical program SPSS version 19 (IBM Corporation, Somers, NY).

\section{RESULTS}

\section{Antagonistic activity of marine-derived fungi}

Twenty marine-derived fungi were selected and identified to species based on morphological and ITS gene analysis and their gene sequences were submitted to Genbank (Table 1). Results of their antagonistic activity against the ten plant pathogenic fungi in the dual cultures on PDA plates are shown in Table 2. Seven of these pathogenic fungi belonged to Ascomycetes (Alternaria brassicicola, Bipolaris oryzae, Colletotrichum capsici, C. gloeosporioides, Fusarium oxysporum, Lasiodiplodia theobromae and Pyricularia oryzae), one to Oomycetes (Phytophthora palmivora) and two to Agonomycetes (Rhizoctonia solani and Sclerotium rolfsii).

Trichoderma asperellum (KUFA 0677) and T. harzianum (KUFA 0689) displayed the highest effect against all plant pathogenic fungi,causing more than $60.65 \%$ mycelial growth inhibition, and they caused $100 \%$ inhibition of $C$. gloeosporioides and $P$. palmivora by overgrowing colonies of these pathogens.

Values in a column followed by the same letter are not significantly different at $p<0.05$, when analyzed using Duncan's multiple range test of One-Way ANOVA.

The results on the antagonistic effects of the rest of the selected marine-derived fungi against plant pathogenic fungi belonging to Ascomycetes revealed that ten of the tested fungi displayed potent (> 50\% inhibition) antagonistic effect against at least one pathogen belonging to this class. Five fungi, namely $A$. xenocordella (KUFA1018), E. nidulans(KUFA0031), H. avellanea (KUFA0450), N. vasinfecta var. vasinfecta (KUFA1004) and T. stipitatus (KUFA0207) exhibited effective mycelial growth inhibition against $A$. brassicicola, C. capsici and $P$. oryzae with values ranging from 50.37 to $66.30 \%$. Meanwhile, $E$. nidulans (KUFA0031), N. fischeri (KUFA0107) and N. pseudofischeri (KUFA0061)also displayed potent antagonistic effect against $B$. oryzae,causing 62$68 \%$ mycelial growth inhibition.

Moreover, A. xenocordella (KUFA1018) and $N$. pseudofischeri (KUFA0061) showed a moderate inhibitory effect, causing 54-55\% mycelial growth inhibition of $F$. oxysporum. Additionally, E. nidulans (KUFA0031) and $M$. verrucaria (KUFA0192) showed effective action against the mycelial growth of $L$. theobromae, with inhibition values of $60-64 \%$.

Besides Trichoderma species, the other marine-derived fungi showed a weak effect, causing mycelial growth inhibition of $P$. palmivora with a value lower than $50 \%$, and only E. nidulans (KUFA0031) exhibited a potent antagonistic effect against $R$. solani and $S$. rolfsii, causing mycelial growth inhibitions of 60.37 and $55.57 \%$, respectively. Interestingly, six out of the twenty marine-derived fungi showed antagonistic activity against the tested plant pathogenic fungi by forming zones of inhibition although they caused mycelial growth inhibition lower than 50\%. T. tratensis (KUFA0091) displayed the 
Chalearmsrimuang et al. J Pure Appl Microbiol, 13(1), 209-223 | March 2019 | DOI 10.22207/JPAM.13.1.22

Table 2. Antagonistic effects of marine-derived fungi on ten plant pathogenic fungi in dual cultures on PDA

\begin{tabular}{|c|c|c|c|c|c|c|c|c|c|c|}
\hline \multirow{2}{*}{$\begin{array}{l}\text { Marine- } \\
\text { derived } \\
\text { fungus }\end{array}$} & \multirow[b]{2}{*}{$A B^{*}$} & \multirow[b]{2}{*}{$\mathrm{BO}$} & \multirow[b]{2}{*}{ CG } & \multicolumn{6}{|c|}{ \% Mycelial growth inhibition } & \multirow[b]{2}{*}{ SR } \\
\hline & & & & $\mathrm{CC}$ & FO & LT & PP & $\mathrm{PO}$ & RS & \\
\hline $\begin{array}{l}\text { Arthrinium } \\
\text { xenocordella }\end{array}$ & $65.19 d$ & $42.59 \mathrm{ij}$ & $45.93 c$ & $57.41 \mathrm{e}$ & $54.81 \mathrm{c}$ & $29.63 \mathrm{~g}$ & $39.26 \mathrm{e}$ & $62.59 \mathrm{~d}$ & Ol & $11.11 \mathrm{f}-\mathrm{h}$ \\
\hline $\begin{array}{l}\text { Emericella } \\
\text { foveolata }\end{array}$ & $49.17 i$ & $44.17 i$ & 37.41gh & $50.74 f$ & 43.70ef & 01 & $36.67 f$ & $48.06 \mathrm{~g}$ & Ol & 3.70hi \\
\hline $\begin{array}{l}\text { Emericella } \\
\text { nidulans }\end{array}$ & $55.57 \mathrm{~g}$ & $68.89 c$ & $44.44 \mathrm{~cd}$ & $51.85 f$ & $40.21 \mathrm{~h}$ & $64.8 \mathrm{a} 1$ & $39.44 \mathrm{e}$ & $56.67 e$ & $60.37 b$ & $55.57 a$ \\
\hline $\begin{array}{l}\text { Emericella } \\
\text { rugulosa }\end{array}$ & $44.44 \mathrm{jk}$ & 39.261 & 37.78gh & $39.30 i$ & $36.34 \mathrm{ij}$ & 01 & $29.26 \mathrm{~h}$ & $47.41 \mathrm{~g}$ & 이 & $19.26 d-f$ \\
\hline $\begin{array}{l}\text { Emericella } \\
\text { variecolor }\end{array}$ & $58.89 f$ & $51.48 \mathrm{~g}$ & $36.66 \mathrm{~h}$ & $43.70 \mathrm{~h}$ & $37.40 \mathrm{i}$ & 01 & $44.44 \mathrm{c}$ & $42.97 i$ & $19.4 \mathrm{~h} 4$ & Oi \\
\hline $\begin{array}{l}\text { Eurotium } \\
\text { chevalieri }\end{array}$ & $45.00 \mathrm{jk}$ & $32.56 \mathrm{~m}$ & $22.78 j$ & $31.11 \mathrm{k}$ & 30.781 & $30.5 \mathrm{~g} 6$ & $21.74 j$ & $39.22 \mathrm{j}$ & $31.89 f$ & $22.2 \mathrm{~d} 2$ \\
\hline $\begin{array}{l}\text { Hamigera } \\
\text { avellanea }\end{array}$ & $64.44 d$ & $54.82 f$ & $48.89 b$ & $58.52 \mathrm{de}$ & $42.96 \mathrm{fg}$ & $34.74 f$ & $41.85 d$ & $66.30 c$ & $14.82 \mathrm{i}$ & $11.11 \mathrm{f}-\mathrm{h}$ \\
\hline $\begin{array}{l}\text { Hamigera } \\
\text { terricola }\end{array}$ & 49.74hi & $46.67 \mathrm{~h}$ & $45.18 c$ & $44.81 \mathrm{~h}$ & $44.44 \mathrm{ef}$ & $23.31 i$ & $27.22 \mathrm{i}$ & $45.13 \mathrm{~h}$ & 01 & 12.17e-g \\
\hline $\begin{array}{l}\text { Geosmithia } \\
\text { lavendula }\end{array}$ & $48.89 i$ & 39.221 & $37.78 \mathrm{gh}$ & $35.21 \mathrm{j}$ & $35.22 \mathrm{j}$ & $41.11 \mathrm{c}$ & $41.48 d$ & $38.85 j$ & $32.47 f$ & $0 \mathrm{i}$ \\
\hline $\begin{array}{l}\text { Myrothecium } \\
\text { verrucaria }\end{array}$ & $48.80 \mathrm{i}$ & $46.67 \mathrm{~h}$ & $48.15 b$ & $45.25 h$ & $32.96 \mathrm{k}$ & $60.00 \mathrm{~b}$ & $44.82 c$ & $48.89 \mathrm{~g}$ & $43.33 c$ & $4.8 \mathrm{~g}-\mathrm{i} 2$ \\
\hline $\begin{array}{l}\text { Neocosmo- } \\
\text { spora } \\
\text { vasinfecta var. } \\
\text { vasinfecta }\end{array}$ & $55.56 \mathrm{~g}$ & 43.70j & $42.22 \mathrm{e}$ & $50.37 f$ & $45.18 \mathrm{e}$ & $26.30 \mathrm{~h}$ & $29.63 \mathrm{~h}$ & $54.44 f$ & $27.76 \mathrm{~g}$ & $22.22 d$ \\
\hline $\begin{array}{l}\text { Penicillium } \\
\text { aculeatum }\end{array}$ & $48.11 \mathrm{i}$ & $43.28 \mathrm{i}$ & $33.14 \mathrm{i}$ & $35.09 j$ & $37.00 \mathrm{i}$ & $15.62 \mathrm{k}$ & $34.74 \mathrm{~g}$ & 29.871 & $10.31 \mathrm{k}$ & $0 \mathrm{i}$ \\
\hline $\begin{array}{l}\text { Neosartorya } \\
\text { fischeri }\end{array}$ & $43.33 k$ & $62.59 d$ & $40.37 f$ & $60.00 \mathrm{~d}$ & 44.41ef & $22.08 \mathrm{i}$ & $41.85 d$ & $45.64 h$ & $28.51 \mathrm{~g}$ & $0 \mathrm{i}$ \\
\hline $\begin{array}{l}\text { Neosartorya } \\
\text { pseudofischeri }\end{array}$ & $67.03 c$ & $60.91 \mathrm{e}$ & $45.58 c$ & $47.63 \mathrm{~g}$ & $55.18 \mathrm{c}$ & $42.37 c$ & $48.69 \mathrm{~b}$ & $47.81 \mathrm{~g}$ & $37.03 e$ & $47.19 \mathrm{~b}$ \\
\hline $\begin{array}{l}\text { Neosartorya } \\
\text { quadricincta }\end{array}$ & $68.52 \mathrm{c}$ & $40.74 \mathrm{kl}$ & 42.92de & $58.52 \mathrm{de}$ & $42.59 \mathrm{fg}$ & $19.26 \mathrm{j}$ & $43.70 c$ & $56.30 \mathrm{e}$ & $40.37 d$ & $35.93 c$ \\
\hline $\begin{array}{l}\text { Neosartorya } \\
\text { tsunodae }\end{array}$ & $45.57 j$ & $41.32 \mathrm{jk}$ & $36.07 \mathrm{~h}$ & $39.58 \mathrm{i}$ & 41.38gh & $20.11 j$ & 40.36de & $35.67 k$ & $12.36 \mathrm{j}$ & $10.87 f-h$ \\
\hline $\begin{array}{l}\text { Talaromyces } \\
\text { tratensis }\end{array}$ & $51.11 \mathrm{~h}$ & $41.11 \mathrm{jk}$ & $38.89 \mathrm{fg}$ & $40.00 i$ & $42.59 \mathrm{fg}$ & $37.03 e$ & $36.67 f$ & $42.96 \mathrm{i}$ & 이 & $15.92 d-f$ \\
\hline $\begin{array}{l}\text { Talaromyces } \\
\text { stipitatus }\end{array}$ & $62.51 \mathrm{e}$ & 47.39h & $48.05 b$ & $65.24 c$ & $49.20 d$ & $39.07 d$ & $48.26 \mathrm{~b}$ & $61.22 d$ & $32.0 f 7$ & 20.14de \\
\hline $\begin{array}{l}\text { Trichoder- } \\
\text { maasperellum }\end{array}$ & $83.33 a$ & $95.68 a$ & $100 a$ & $80.00 \mathrm{~b}$ & $72.96 b$ & $60.65 b$ & $100 a$ & $81.15 b$ & $71.0 \mathrm{a} 0$ & $61.48 a$ \\
\hline $\begin{array}{l}\text { Trichoderm- } \\
\text { aharzianum }\end{array}$ & $80.25 b$ & $92.37 b$ & $100 a$ & $92.11 a$ & $81.12 a$ & $59.31 b$ & $100 a$ & $90.35 a$ & $70.25 a$ & $68.32 a$ \\
\hline
\end{tabular}

${ }^{*} \mathrm{AB}=$ Alternaria brassicicola, $\mathrm{BO}=$ Bipolaris oryzae, $\mathrm{CC}=$ Colletotrichum capsici, $\mathrm{CG}=\mathrm{C}$. gloeosporiodes, $\mathrm{FO}=$ Fusarium oxysporum, $\mathrm{LT}$

$=$ Lasiodiplodia theobromae, $\mathrm{PP}=$ Phytophthora palmivora, $\mathrm{PO}=$ Pyricularia oryzae, $\mathrm{RO}=$ Rhizoctonia oryzae, $\mathrm{SR}=$ Sclerotium rolfsii 
strongest activity with formation of the widest zone of inhibition, 1.2 to $2.2 \mathrm{~cm}$ in width, against A. brassicicola, B. oryzae, $L$. theobromae and $P$. palmivora. In addition, A. xenocordella,E. rugulosa (KUFA1002), E. foveolata (KUFA1003), $N$. vasinfecta var. vasinfecta (KUFA1004) and $N$. pseudofischeri (KUFA0061) showed antagonistic activity by forming zones of inhibition 0.5-1.2 $\mathrm{cm}$ in width against some plant pathogenic fungi belonging to Ascomycetes (Fig. 1).

Antifungal activity of marine-derived fungi The result of testing the antifungal
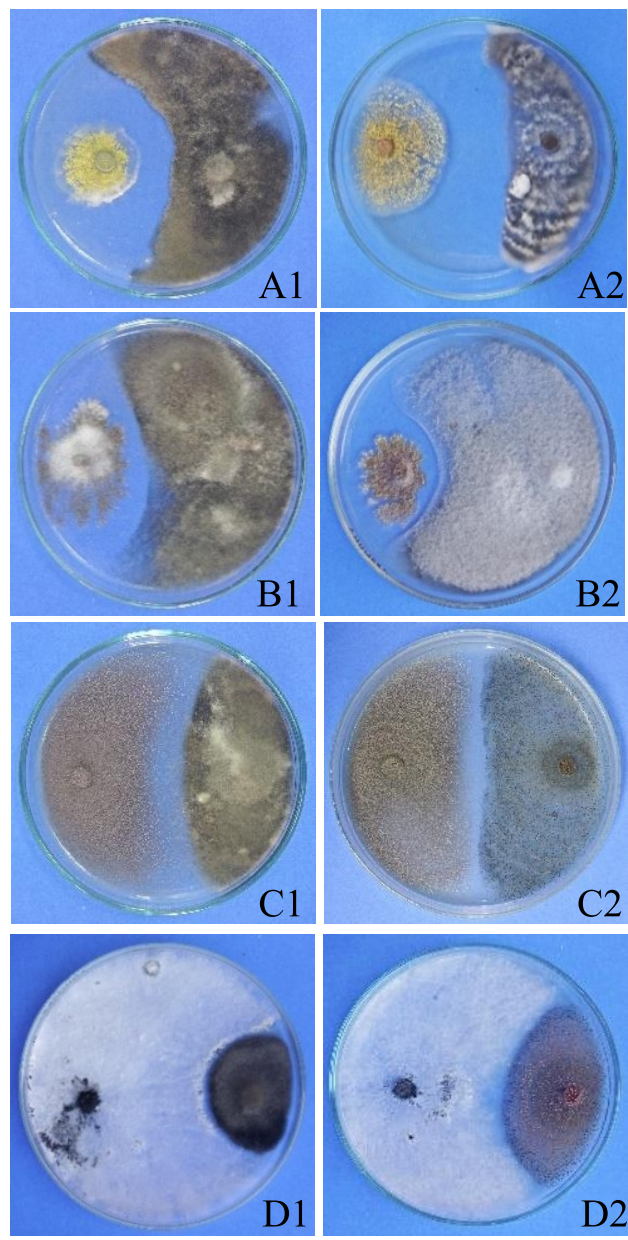

D1

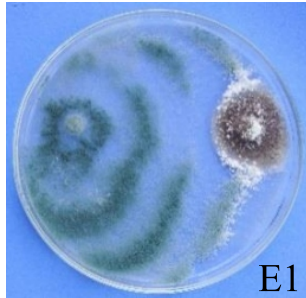

E1
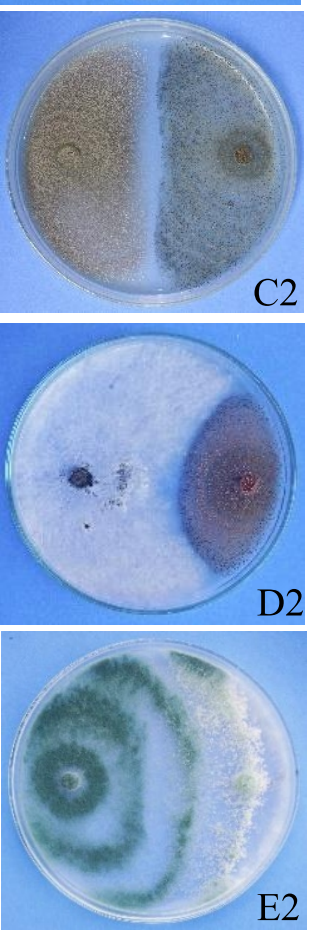
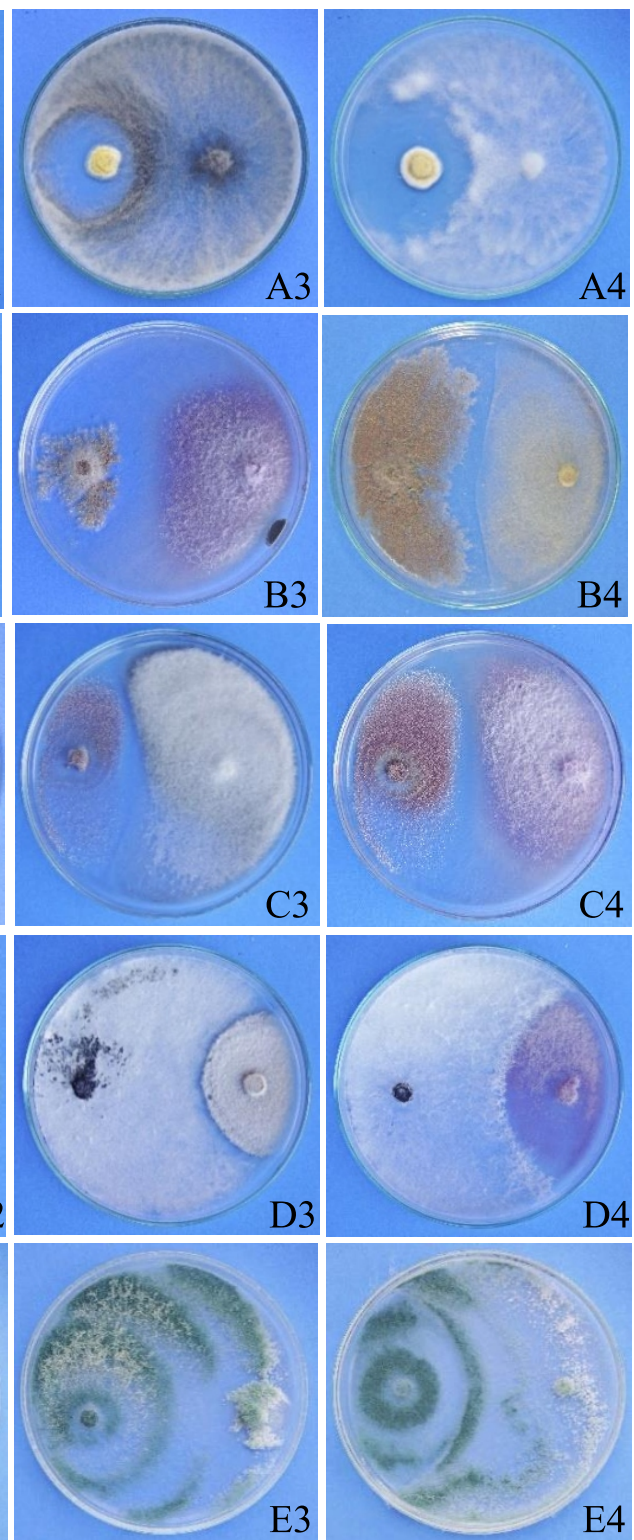

Fig. 1. Antagonistic effects of marine-derived fungi (left) on plant pathogenic fungi (right) in dual cultures on PDA plates.

A. Talaromyces tratensis KUFA0091 vs A. brassicicola (A1), B. oryzae (A2), L. theobromae(A3),P. palmivora(A4)

B. Emericella rugulosa KUFA1002 vs A. brassicicola (B1), C. gloeosporiodes (B2), F. oxysporum (B3), P. oryzae (B4) C. Neocosmospora vasinfecta var. vasinfecta KUFA1004 vs A. brassicicola (C1), C. capsici (C2), C. gloeosporiodes (C3), F. oxysporum(C4) D. Arthrinium xenocordella KUFA1018 vs A. brassicicola (D1), C. capsici (D2), C. gloeosporiodes (D3),F. oxysporum(D4) E. Trichoderma harzianum KUFA0677 vs A. brassicicola (E1), P. palmivora (E2), P. oryzae (E3),R. oryzae (E4) 
activity of marine-derived fungi crude ethyl acetate extracts against the ten plant pathogenic fungi revealed that the crude extracts displayed increased effect against plant pathogens when the concentration increased (Table 3). At the highest dose tested, $10 \mathrm{~g} / \mathrm{L}$, all fungal extracts except E. chevalieri (KUFA0464), G. lavendula (KUFA0319), and N. pseudofischeri (KUFA0061)

Table 3. Antifungal effects of marine-derived fungal extracts on ten plant pathogenic fungi by using the dilution method.

\begin{tabular}{|c|c|c|c|c|c|c|c|c|c|c|}
\hline \multirow{3}{*}{$\begin{array}{l}\text { Marine- } \\
\text { derived } \\
\text { fungal } \\
\text { extract }\end{array}$} & \multicolumn{10}{|c|}{$\%$ Mycelial growth inhibition at different concentrations (g/L) } \\
\hline & \multicolumn{2}{|c|}{$A B^{*}$} & \multicolumn{2}{|c|}{$\mathrm{BO}$} & \multicolumn{2}{|c|}{ CC } & \multicolumn{2}{|l|}{ CG } & \multicolumn{2}{|l|}{ FO } \\
\hline & 10 & 1 & 10 & 1 & 10 & 1 & 10 & 1 & 10 & 1 \\
\hline $\begin{array}{l}\text { Arthrinium } \\
\text { xenocordella }\end{array}$ & $100 \mathrm{a}$ & $37.78 n$ & $100 \mathrm{a}$ & 38.520 & $100 a$ & $15 \mathrm{k}$ & $40 \mathrm{j}$ & $\mathrm{Ou}$ & $54.72 i$ & Or \\
\hline $\begin{array}{l}\text { Emericella } \\
\text { foveolata }\end{array}$ & $100 \mathrm{a}$ & $28.61 p$ & $100 \mathrm{a}$ & $49.44 j$ & $100 a$ & $17.22 \mathrm{k}$ & $55 \mathrm{~h}$ & $31.67 \mathrm{Im}$ & $100 a$ & $16.94 p$ \\
\hline $\begin{array}{l}\text { Emericella } \\
\text { nidulans }\end{array}$ & $44.441 \mathrm{~m}$ & $21.11 u$ & $36.66 p$ & $0 x$ & $51.67 e$ & $18.61 \mathrm{k}$ & $32.22 \mathrm{klm}$ & $11.11 p$ & $17.40 p$ & Or \\
\hline $\begin{array}{l}\text { Emericella } \\
\text { rugulosa }\end{array}$ & $75.50 \mathrm{de}$ & $63.06 i$ & $100 a$ & $55.28 \mathrm{i}$ & $100 a$ & $0 m$ & $100 a$ & $34.72 \mathrm{k}$ & $100 a$ & 250 \\
\hline $\begin{array}{l}\text { Emericella } \\
\text { variecolor }\end{array}$ & $100 a$ & $78.14 d$ & $100 a$ & $0 x$ & $100 a$ & $72.77 d$ & $100 a$ & $78.88 d$ & $100 a$ & $83.70 c$ \\
\hline $\begin{array}{l}\text { Eurotium } \\
\text { chevalieri }\end{array}$ & 35.170 & Os & $29.18 \mathrm{~s}$ & $11.76 \mathrm{w}$ & $24.22 \mathrm{j}$ & $15.76 \mathrm{k}$ & $30.251 \mathrm{~m}$ & 12.31op & $37.14 \mathrm{~m}$ & $10.32 q$ \\
\hline $\begin{array}{l}\text { Hamigera } \\
\text { avellanea }\end{array}$ & $66.66 \mathrm{~h}$ & $43.331 \mathrm{~m}$ & $63.04 j$ & $23.70 \mathrm{t}$ & $100 a$ & $23.70 \mathrm{j}$ & $100 a$ & $30 \mathrm{~m}$ & $66.66 \mathrm{e}$ & $35.25 \mathrm{~m}$ \\
\hline $\begin{array}{l}\text { Hamigera } \\
\text { terricola }\end{array}$ & $60.12 j$ & Os & $60.45 \mathrm{~h}$ & $35.47 q r$ & $100 a$ & $30.04 i$ & $72.59 \mathrm{e}$ & $39.25 j$ & $74.10 d$ & $45.32 \mathrm{k}$ \\
\hline $\begin{array}{l}\text { Geosmithia } \\
\text { lavendula }\end{array}$ & $54.10 \mathrm{k}$ & Os & $71.42 \mathrm{e}$ & $0 x$ & $74.12 d$ & $14.36 \mathrm{k}$ & $68.21 f$ & $\mathrm{Ou}$ & $57.84 \mathrm{fh}$ & $31.22 n$ \\
\hline $\begin{array}{l}\text { Myrothecium } \\
\text { verrucaria }\end{array}$ & $100 a$ & $44.41 \mathrm{~lm}$ & $100 a$ & $100 a$ & $100 a$ & $72.72 d$ & $100 a$ & $73.70 \mathrm{e}$ & $100 a$ & $87.77 b$ \\
\hline $\begin{array}{l}\text { Neocosmospora } \\
\text { vasinfecta var. } \\
\text { vasinfecta }\end{array}$ & $78.89 d$ & $67.78 \mathrm{~h}$ & 42.781 & $44.72 \mathrm{k}$ & $100 a$ & $46.94 f$ & $68.33 f$ & $45.22 i$ & $49.72 j$ & $38.06 \mathrm{~m}$ \\
\hline $\begin{array}{l}\text { Penicillium } \\
\text { aculeatum }\end{array}$ & $82.14 c$ & $24.11 q$ & $35.36 q r$ & $0 x$ & $87.61 b$ & 48.30ef & $84.64 c$ & $32.56 \mathrm{klm}$ & $58.97 f$ & Or \\
\hline $\begin{array}{l}\text { Neosartorya } \\
\text { fischeri }\end{array}$ & $100 a$ & $42.32 \mathrm{~m}$ & $100 a$ & $0 x$ & $100 a$ & $18.50 \mathrm{k}$ & $100 a$ & $20.20 n$ & $100 a$ & $35.25 \mathrm{~m}$ \\
\hline $\begin{array}{l}\text { Neosartorya } \\
\text { pseudofischeri }\end{array}$ & $75.50 \mathrm{e}$ & $45.43 \mid$ & $80 c$ & $40.75 \mathrm{~m}$ & $82.94 c$ & $35.47 \mathrm{~h}$ & $95.50 \mathrm{~b}$ & $20.59 n$ & 55.41hi & $10.50 q$ \\
\hline $\begin{array}{l}\text { Neosartorya } \\
\text { quadricincta }\end{array}$ & 35.51on & Os & $21.18 u$ & $0 x$ & $5.73 i$ & $0 m$ & $\mathrm{Ou}$ & $\mathrm{Ou}$ & $15.32 p$ & Or \\
\hline $\begin{array}{l}\text { Neosartorya } \\
\text { tsunodae }\end{array}$ & $100 a$ & $26.67 p$ & $65.83 f$ & $34.17 r$ & $100 a$ & $16.39 \mathrm{k}$ & $100 a$ & 14.440 & $36.39 \mathrm{~m}$ & Or \\
\hline $\begin{array}{l}\text { Talaromyces } \\
\text { tratensis }\end{array}$ & $100 a$ & $37.78 n$ & $100 a$ & $55.92 i$ & $100 a$ & $18.61 \mathrm{k}$ & $100 a$ & $4.44 q$ & $100 a$ & $7.50 u$ \\
\hline $\begin{array}{l}\text { Talaromyces } \\
\text { stipitatus }\end{array}$ & $28.33 p$ & $5.56 r$ & $87.78 b$ & $40.02 \mathrm{mn}$ & $45 f$ & 4.17lm & $38.33 \mathrm{j}$ & $\mathrm{Ou}$ & $30.83 n$ & Or \\
\hline $\begin{array}{l}\text { Trichoderma- } \\
\text { asperellum }\end{array}$ & $88.89 \mathrm{~b}$ & Os & $77.22 d$ & $44.44 k$ & $80.28 c$ & $0 m$ & $32.96 \mathrm{kl}$ & $\mathrm{Ou}$ & 41.111 & $6.94 u$ \\
\hline $\begin{array}{l}\text { Trichoderma- } \\
\text { harzianum }\end{array}$ & $70.48 f$ & $20.17 u$ & $65.42 f$ & $14.37 v$ & $100 a$ & $15 \mathrm{k}$ & $45.87 i$ & $\mathrm{Ou}$ & $31.89 n$ & Or \\
\hline
\end{tabular}


Table 3. Continued

\begin{tabular}{|c|c|c|c|c|c|c|c|c|c|c|}
\hline Marine- & & & \% Mycel & lial grow & th inhibiti & on at diffe & rent conce & itrations & $(\mathrm{g} / \mathrm{L})$ & \\
\hline derived & LT & & $\mathrm{PP}$ & & $\mathrm{PO}$ & & RS & & SR & \\
\hline $\begin{array}{l}\text { fungal } \\
\text { extract }\end{array}$ & 10 & 1 & 10 & 1 & $\overline{10}$ & $\overline{1}$ & $\overline{10}$ & $\overline{1}$ & $\overline{10}$ & 1 \\
\hline $\begin{array}{l}\text { Arthrinium } \\
\text { xenocordella }\end{array}$ & $100 a$ & Oj & $100 a$ & $19.63 \mid$ & $100 a$ & Oj & $100 a$ & $0 m$ & $100 a$ & $9.17 n$ \\
\hline $\begin{array}{l}\text { Emericella } \\
\text { foveolata }\end{array}$ & $67.22 d$ & Oj & $100 a$ & $39.17 \mathrm{~h}$ & $100 a$ & $18.89 i$ & 50.00ef & $6.67 i$ & 51.48 ef & $16.39 \mathrm{~m}$ \\
\hline $\begin{array}{l}\text { Emericella } \\
\text { nidulans }\end{array}$ & $100 a$ & $55.78 \mathrm{e}$ & $100 a$ & $57.44 \mathrm{e}$ & $100 a$ & $77.22 b c$ & $57.40 d$ & $0 m$ & $100 a$ & $49.44 f$ \\
\hline $\begin{array}{l}\text { Emericella } \\
\text { rugulosa }\end{array}$ & $100 a$ & $36.39 \mathrm{~h}$ & $100 a$ & $64.44 d$ & $100 a$ & $34.07 \mathrm{~h}$ & $100 a$ & $12.22 \mathrm{k}$ & $100 a$ & $29.44 j$ \\
\hline $\begin{array}{l}\text { Emericella } \\
\text { variecolor }\end{array}$ & $100 a$ & Oj & $100 a$ & $0 m$ & $100 a$ & Oj & $100 a$ & $0 m$ & $100 a$ & 0o \\
\hline $\begin{array}{l}\text { Eurotium } \\
\text { chevalieri }\end{array}$ & oj & Oj & $0 m$ & $0 m$ & $0 \mathrm{j}$ & Oj & $10.12 \mathrm{k}$ & $0 m$ & $21.35 I$ & 0o \\
\hline $\begin{array}{l}\text { Hamigera } \\
\text { avellanea }\end{array}$ & $100 a$ & $5.55 \mathrm{j}$ & $100 a$ & $0 m$ & $100 a$ & $33.33 \mathrm{~h}$ & $100 a$ & $66.66 c$ & $100 a$ & $25.92 k$ \\
\hline $\begin{array}{l}\text { Hamigera } \\
\text { terricola }\end{array}$ & $100 a$ & Oj & 55.55ef & $30.12 \mathrm{j}$ & $51.14 f$ & 12.17ij & $85.40 \mathrm{~b}$ & $22.81 \mathrm{j}$ & $74.35 c$ & $32.17 i$ \\
\hline $\begin{array}{l}\text { Geosmithia } \\
\text { lavendula }\end{array}$ & $24.92 \mathrm{i}$ & Oj & $64.65 d$ & $22.57 \mathrm{k}$ & 62.03de & Oj & $47.25 \mathrm{fh}$ & $0 m$ & $58.22 d$ & 0o \\
\hline $\begin{array}{l}\text { Myrothecium } \\
\text { verrucaria }\end{array}$ & $100 a$ & $100 a$ & $100 a$ & $35.92 i$ & $100 a$ & 52.17ef & $100 a$ & $24.51 j$ & $100 a$ & 0o \\
\hline $\begin{array}{l}\text { Neocosmospora } \\
\text { vasinfecta var. } \\
\text { vasinfecta }\end{array}$ & $68.89 d$ & $25.28 \mathrm{i}$ & $100 a$ & $0 m$ & $68.61 \mathrm{~cd}$ & 52.50ef & 51.67e & $10.00 \mathrm{k}$ & 31.39ij & 0o \\
\hline $\begin{array}{l}\text { Penicillium } \\
\text { aculeatum }\end{array}$ & $97.21 a$ & $32.58 \mathrm{~h}$ & $100 a$ & $56.82 \mathrm{e}$ & $86.21 b$ & $11.25 \mathrm{ij}$ & 47.39fh & $28.00 i$ & $59.21 d$ & 0o \\
\hline $\begin{array}{l}\text { Neosartorya } \\
\text { fischeri }\end{array}$ & Oj & Oj & $100 a$ & $0 m$ & $100 a$ & $33.33 \mathrm{~h}$ & $100 a$ & $45.42 \mathrm{~h}$ & $100 a$ & 53.75e \\
\hline $\begin{array}{l}\text { Neosartorya } \\
\text { pseudofischeri }\end{array}$ & $87.25 b$ & $45.81 f$ & $75.25 c$ & $20.37 \mathrm{kl}$ & $74.22 \mathrm{c}$ & $35.47 \mathrm{~h}$ & $65.40 c$ & $45 \mathrm{~h}$ & $72.57 c$ & 20.351 \\
\hline $\begin{array}{l}\text { Neosartorya } \\
\text { quadricincta }\end{array}$ & Oj & 0j & $86.95 b$ & $36 \mathrm{i}$ & $0 \mathrm{j}$ & 0j & $100 a$ & $0 m$ & $95.56 \mathrm{~b}$ & 00 \\
\hline $\begin{array}{l}\text { Neosartorya } \\
\text { tsunodae }\end{array}$ & $76.94 c$ & Oj & $100 a$ & $20.28 \mathrm{kl}$ & $100 a$ & Oj & $100 a$ & $4.72 \mathrm{i}$ & $100 a$ & $42.22 \mathrm{~h}$ \\
\hline $\begin{array}{l}\text { Talaromyces } \\
\text { tratensis }\end{array}$ & $76.39 c$ & Oj & $100 a$ & $53.33 f$ & $100 a$ & $32.50 \mathrm{~h}$ & $21.94 j$ & $0 m$ & $100 a$ & 0o \\
\hline $\begin{array}{l}\text { Talaromyces } \\
\text { stipitatus }\end{array}$ & $100 a$ & Oj & $100 a$ & $0 m$ & $100 a$ & $15.56 \mathrm{i}$ & $100 a$ & $0 m$ & $100 a$ & 0o \\
\hline $\begin{array}{l}\text { Trichoderma- } \\
\text { asperellum }\end{array}$ & $64.44 d$ & Oj & $100 a$ & $0 m$ & $100 a$ & oj & $100 a$ & $0 m$ & $100 a$ & 0o \\
\hline $\begin{array}{l}\text { Trichoderma- } \\
\text { harzianum }\end{array}$ & 50.11ef & $0 \mathrm{j}$ & $100 a$ & $0 m$ & $100 a$ & $0 \mathrm{j}$ & $100 a$ & $0 m$ & $100 a$ & 0o \\
\hline
\end{tabular}

${ }^{*} \mathrm{AB}=$ Alternaria brassicicola, $\mathrm{BO}=$ Bipolaris oryzae, $\mathrm{CC}=$ Colletotrichum capsici, $\mathrm{CG}=\mathrm{C}$. gloeosporiodes, $\mathrm{FO}=$ Fusarium oxysporum, $\mathrm{LT}=$ Lasiodiplodia theobromae, $\mathrm{PP}=$ Phytophthora palmivora, $\mathrm{PO}=$ Pyricularia oryzae, $\mathrm{RO}=$ Rhizoctonia oryzae, $\mathrm{SR}=$ Sclerotium rolfsii 
extracts exhibited $100 \%$ mycelial growth inhibition of at least two of the plant pathogens tested. $M$. verrucaria (KUFA0192) crude extract displayed the greatest antifungal activity, causing $100 \%$ inhibition against all tested plant pathogens at 10 $\mathrm{g} / \mathrm{L}$ and also complete inhibition of $B$. oryzae and L. theobromae mycelial growth at $1 \mathrm{~g} / \mathrm{L}$.

At $1 \mathrm{~g} / \mathrm{L}$, the crude extracts of seven marine-derived fungi: $E$. nidulans (KUFA0031), $E$. rugulosa (KUFA1002), E. variecolor (KUFA0261),

Table 4. $\mathrm{NaCl}$ tolerance of marine-derived fungi

\begin{tabular}{|c|c|c|c|c|c|c|c|}
\hline \multirow[t]{2}{*}{$\begin{array}{l}\text { Marine-derived } \\
\text { fungus }\end{array}$} & \multicolumn{7}{|c|}{$\begin{array}{l}\text { Mycelial growth of marine-derived fungi on } \\
\text { PDA amended with } \mathrm{NaCl} \text { at different concentrations }\end{array}$} \\
\hline & $0 \%$ & $1 \%$ & $3 \%$ & $5 \%$ & $7 \%$ & $10 \%$ & $15 \%$ \\
\hline $\begin{array}{l}\text { Arthrinium } \\
\text { xenocordella }\end{array}$ & 9 & 9 & 9 & 9 & 9 & $7.2 \pm 0.16$ & $-1 /$ \\
\hline $\begin{array}{l}\text { Emericella } \\
\text { foveolata }\end{array}$ & 9 & 9 & 9 & 9 & $8.4 \pm 1.97$ & $7.84 \pm 0.21$ & $3.4 \pm 0.22$ \\
\hline $\begin{array}{l}\text { Emericella } \\
\text { nidulans }\end{array}$ & 9 & 9 & 9 & 9 & 9 & $6.2 \pm 0.34$ & $3.52 \pm 0.24$ \\
\hline $\begin{array}{l}\text { Emericella } \\
\text { rugulosa }\end{array}$ & 9 & 9 & 9 & 9 & 9 & $5.5 \pm 0.11$ & - \\
\hline $\begin{array}{l}\text { Emericella } \\
\text { variecolor }\end{array}$ & 9 & 9 & 9 & $7.21 \pm 0.59$ & $4.25 \pm 0.74$ & $3.43 \pm 0.89$ & $2.3 \pm 0.18$ \\
\hline $\begin{array}{l}\text { Eurotium } \\
\text { chevalieri }\end{array}$ & $2.34 \pm 0.24$ & $2.64 \pm 0.20$ & $3.28 \pm 0.32$ & $3.38 \pm 0.18$ & $3.46 \pm 0.21$ & $3.14 \pm 0.15$ & $3.27 \pm 0.20$ \\
\hline $\begin{array}{l}\text { Hamigera } \\
\text { avellanea }\end{array}$ & 9 & 9 & 9 & 9 & 9 & $4.56 \pm 0.06$ & - \\
\hline $\begin{array}{l}\text { Hamigera } \\
\text { terricola }\end{array}$ & 9 & 9 & 9 & 9 & $5.5 \pm 0.25$ & $2.9 \pm 0.23$ & - \\
\hline $\begin{array}{l}\text { Geosmithia } \\
\text { lavendula }\end{array}$ & 9 & 9 & 9 & 9 & 9 & $7.54 \pm 0.04$ & $1.57 \pm 0.03$ \\
\hline $\begin{array}{l}\text { Myrothecium } \\
\text { verrucaria }\end{array}$ & 9 & 9 & 9 & $6.54 \pm 1.12$ & $4.5 \pm 0.19$ & - & - \\
\hline $\begin{array}{l}\text { Neocosmospora } \\
\text { vasinfecta var. } \\
\text { vasinfecta }\end{array}$ & 9 & 9 & 9 & 9 & $7.5 \pm 0.58$ & $4.62 \pm 0.29$ & - \\
\hline $\begin{array}{l}\text { Penicillium } \\
\text { aculeatum }\end{array}$ & 9 & 9 & 9 & $5.37 \pm 0.85$ & $1.32 \pm 0.28$ & - & - \\
\hline $\begin{array}{l}\text { Neosartorya } \\
\text { fischeri }\end{array}$ & 9 & 9 & 9 & 9 & 9 & - & - \\
\hline $\begin{array}{l}\text { Neosartorya } \\
\text { pseudofischeri }\end{array}$ & 9 & 9 & 9 & 9 & 9 & - & - \\
\hline $\begin{array}{l}\text { Neosartorya } \\
\text { quadricincta }\end{array}$ & 9 & 9 & 9 & 9 & 9 & - & - \\
\hline $\begin{array}{l}\text { Neosartorya } \\
\text { tsunodae }\end{array}$ & $\begin{array}{l}7.13 \pm 0.57 \\
-\end{array}$ & $\begin{array}{l}6.58 \pm 0.41 \\
-\end{array}$ & $5.67 \pm 0.27$ & $4.36 \pm 0.26$ & $2.07 \pm 0.15$ & & \\
\hline $\begin{array}{l}\text { Talaromyces } \\
\text { tratensis }\end{array}$ & $\begin{array}{l}9 \\
-\end{array}$ & 9 & $5.68 \pm 0.38$ & $3.59 \pm 0.29$ & $2.74 \pm 0.21$ & $1.38 \pm 0.09$ & \\
\hline $\begin{array}{l}\text { Talaromyces } \\
\text { stipitatus }\end{array}$ & 9 & 9 & 9 & $7.08 \pm 0.97$ & $3.64 \pm 0.35$ & - & - \\
\hline $\begin{array}{l}\text { Trichoderma- } \\
\text { asperellum }\end{array}$ & 9 & 9 & 9 & 9 & $6.5 \pm 1.54$ & - & - \\
\hline $\begin{array}{l}\text { Trichoderma- } \\
\text { harzianum }\end{array}$ & 9 & 9 & 9 & 9 & $5.5 \pm 0.87$ & - & - \\
\hline
\end{tabular}

${ }^{1 /}$ No growth was observed. 
N. vasinfecta var. vasinfecta (KUFA1004), N. fischeri (KUFA0107), P. aculeatum (KUFA0201) and $T$. tratensis (KUFA0091) displayed significant antifungal activity against plant pathogenic fungi,causing more than $50 \%$ inhibition of at least one plant pathogenic fungus. Among them, E. variecolor (KUFA0261) showed great inhibition (72-83\%) of the mycelial growth of $A$. brassicicola, C. capcisi, C. gloeosporioides and F. oxysporum whereas E. rugulosa (KUFA1002)extract exhibited an antifungal effect on $A$. brassicicola, $B$. oryzae and $P$. palmivora of $55-64 \%$ and $E$. nidulans extract caused $55-72 \%$ inhibition of $L$. theobromae, $P$. palmivora and $P$. oryzae. Furthermore, $T$. tratensis (KUFA0091) extract displayed promising antifungal effect against the mycelial growth of $B$. oryzae and P. palmivora causing 53-55\% inhibition at $1 \mathrm{~g} / \mathrm{L}$. $P$. aculeatum (KUFA0201)and $N$. fischeri(KUFA0107) extracts exhibited 56 and $54 \%$ inhibition of mycelial growth of P. palmivora and S. rolfsii, respectively.

Values in two columns of each pathogen followed by the same letter are not significantly different at $p<0.05$, when analyzed using Duncan's multiple range test of One-Way ANOVA.

\section{Halo tolerance of marine-derived fungi}

The result of testing the salt tolerance of marine-derived fungi on PDA amended with $\mathrm{NaCl}$ at different concentrations is shown in Table 4. All marine-derived fungi exhibited $\mathrm{NaCl}$ tolerance, being able to grow on PDA amended with $\mathrm{NaCl}$ up to $7 \%$, but none of them were able to grow on PDA amended with $\mathrm{NaCl}$ at $20 \%$ and $25 \%$. Five of them showed high tolerance to $\mathrm{NaCl}$, being able to grow slowly on PDA amended with $\mathrm{NaCl}$ at $15 \%$,and another six species were able to grow at $10 \% \mathrm{NaCl}$ concentration. The effects of $\mathrm{NaCl}$ on fungal growth observed included inhibition of fungal growth compared with the controls when $\mathrm{NaCl}$ 's concentrations were increased except in E. chevalieri (KUFA0464). Moreover, the teleomorphic species of Penicillium and Aspergillus exhibited only the anamorphic state, producing conidiophores without cleistothecial formation.

\section{DISCUSSION}

The antagonistic activity of the selected twenty marine-derived fungi against plant pathogenic fungi and their halo tolerance were evaluated. The preliminary results of the dual culture assay showed that among the twenty marine-derived fungi tested, Trichoderma species, T. asperellum and T. harzianum exhibited higher antagonistic effect against all the plant pathogens than the other marine-derived fungi since they caused percentages of mycelial growth inhibition in the range $59.31-100 \%$. Both Trichoderma species showed antagonistic effects on plant pathogenic fungi via overgrowing colonies of plant pathogenic fungi. Trichoderma species are a common genus in various hosts and are the well-known BCAs which act by means of various mechanisms against plant pathogenic fungi including mycoparasitism and producing cell-wall degrading enzymes and antifungal substances ${ }^{36-37}$. According with our results, for example, Trichoderma strains which were isolated from the Mediterranean sponge, Psammocinia sp. collected in Israel showed coiling mycoparasitism on mycelium of Fusarium equiseti when tested on PDA dual cultures ${ }^{18}$ and Trichoderma atroviride and T. asperelloides extracts effectively reduced the incidence of $R$. solani damping-off disease of beans and also induced defense responses in cucumber seedlings against Pseudomonas syringae pv. lachrimans ${ }^{33}$. It is without a doubt that Trichoderma strains are great antagonists and diverse in habitats even in marine environments. Besides, the salt tolerant strains of Trichoderma have been investigated for their activity against plant pathogens to develop BCAs applied in crop protection for application in arid and saline soil areas $33,38,39$.

In contrast, Trichoderma crude extracts showed high antifungal effect on plant pathogens only at the highest concentration, $10 \mathrm{~g} / \mathrm{L}$, and they displayed low to medium activity against all the tested plant pathogens at $1 \mathrm{~g} / \mathrm{L}$. These results accord with a previously reported of the antifungal effect of an entomopathogenic strain of Trichoderma atroviride was lowest against the olive pathogens, Verticillium dahlia, Phytopthora megasperma and Phytopthora inundata ${ }^{40}$.

However, six out of the twenty marinederived fungi displayed antagonistic effects by forming zones of inhibition against the tested plant pathogenic fungi although the average percentage of their mycelial growth inhibition was lower than $50 \%$. For example, Talaromyces tratensis (KUFA 0091) displayed the strongest activity, forming the widest zone of inhibition, 1.2 to $2.2 \mathrm{~cm}$ in width, 
against $A$. brassicicola, $B$. oryzae, L. theobromae and P. Palmivora (Fig. 1). Moreover, E.rugulosa (KUFA1002), E. foveolata (KUFA1003), N. vasinfecta var. vasinfecta (KUFA1004) and Neosartorya pseudofischeri showed antagonistic activity by forming zones of inhibition in the range of 0.5-1.2 $\mathrm{cm}$ in width against some phytopathogenic fungi belonging to Ascomycetes (Fig. 1). These findings showed that these marine-derived fungi produced and released antifungal substances which inhibited the growth of the plant pathogenic fungi.

The results of the dilution plate assay confirmed their production of antifungal substances. Crude extracts of eight marine-derived fungi in the genera Emericella, Myrothecium, Neocosmospora, Penicillium and Talaromyces displayed great antifungal activity against the plant pathogenic fungi at a low concentration of $1 \mathrm{~g} / \mathrm{L}$. The crude extract of $M$. verrucaria showed the best antifungal activity, causing more than $52-100 \%$ inhibition of five of the tested plant pathogenic fungus species at $1 \mathrm{~g} / \mathrm{L}$. This result is in accordance with a previous study which reported that crude ethyl acetate extract of Myrothecium sp. associated with the marine sponge, Axinella sp., was a potential producer of antifungal compounds against Sclerotinia sclerotiorum, a causal agent of stem rot in various crops ${ }^{41}$. Meanwhile, the crude extracts of three Emericella species including $E$. nidulans, E. rugulosa and E. variecolor showed high inhibition of the mycelial growth of eight of the tested plant pathogenic fungi at $1 \mathrm{~g} / \mathrm{L}$. Among them, E. variecolor extract displayed the greatest inhibition, causing $72-83 \%$ inhibition of A. brassicicola, C. capcisi, C. gloeosporioides and $F$. oxysporum, whereas $E$. rugulosa extract exhibited antifungal effects on $A$. brassicicola, B. oryzae and P. palmivora of 55-64\%, and E. nidulans extract caused $55-72 \%$ inhibition of $L$. theobromae, $P$. palmivora and $P$. oryzae. Emericella species are common soil fungi and have been reported as antibiosis producers against plant pathogens. For example, crude extracts of soil strains of $E$. rugulosa and $E$. nidulans showed great antifungal effects against $F$. oxysporum f.sp. lycopersici and C. gloeosporiodes with $\mathrm{ED}_{50}$ values 5.98 and 1000 $\mu \mathrm{g} / \mathrm{mL}$, respectively ${ }^{42-43}$. A few studies reported the antifungal effects of $E$. variecolor extracts on plant pathogens. For instance, crude extracts of soil strains of $E$. nidulans, E. rugulosa and $E$. variecolor were evaluated the antifungal activity and they inhibited by $45-63 \%$ the mycelial growth of $A$. brassicicola, Curvularia lunata, C. capsici, $C$. gloeosporioides, F. oxysporum, Helminthosporium sp., Pestalotiopsis sp. and P. palmivora in vitro. When compared with our results, the extract of a marine strain of $E$. variecolor displayed higher antifungal activity against plant pathogens than that of the extract obtained from a soil strain, for it exhibited $72-83 \%$ inhibition of $A$. brassicicola, $C$. capcisi, C. gloeosporioides and F. oxysporum ${ }^{44}$.

The results in this study also showed that T. tratensis crude extract displayed a promising antifungal effect against the mycelial growth of $B$. oryzae and $P$. palmivora, causing 53-55\% inhibition at $1 \mathrm{~g} / \mathrm{L}$. This is similar to our previous study in which we reported that the crude ethyl acetate extract of Talaromyces trachyspermus (KUFA 0021) exhibited the most effective mycelial growth inhibition of $A$. brassicicola, C. capsici, H. maydis, Pythium aphanidermatum, $R$. solani and $S$. rolfsii with IC I0 $_{50}$ values of $100-186 \mathrm{ppm}$ and displayed total inhibition of mycelial growth on all plant pathogenic fungi at the highest concentration tested, $10 \mathrm{~g} / \mathrm{L}^{10}$.

The results of this study also reveal that at $1 \mathrm{~g} / \mathrm{L}, P$. aculeatum and $N$. fischeri extracts exhibited 56 and $54 \%$ inhibition of mycelial growth of $P$. palmivora and $S$. rolfsii, respectively. Similar to our findings, Shen et al. ${ }^{32}$ reported the antimicrobial activity of marine-derived Penicillium oxalicum strain $0312 \mathrm{~F}$ crude extract, which displayed strong antifungal activity against $A$. brassicicola and $F$. graminearum. In addition, the antifungal activity of Penicillium citrinum isolated from a marine sponge, Callyspongia diffusa, collected in the Gulf of Mannar, on the southeast coast of India ${ }^{45}$. Penicillium citrinum crude extract also displayed strong antifungal activity against nine plant pathogenic fungi, including Alternaria alternata, Botrytis cinerea, Cercospora theae, Fusarium udum, F. oxysporum, Macrophomina phaseolina, Poria hypolateritia, Phomopsis thae and $R$. solani. The result of this study also reveals that $N$. vasinfecta var. vasinfecta extract exhibited 52-67\% inhibition of mycelial growth of A. brassicicola and P. oryzae at $1 \mathrm{~g} / \mathrm{L}$; however, it is not suitable for development as a BCA since it was reported as a causal agent of soybean stem $\operatorname{rot}^{46,47}$. 
The result of testing the halo tolerance of the marine-derived fungi on PDA amended with $\mathrm{NaCl}$ at different concentrations showed that all marine-derived fungi exhibited $\mathrm{NaCl}$ tolerance, being able to grow on PDA amended with $\mathrm{NaCl}$ up to $7 \%$. The tested genera Emericella, Hamigera and Geosmithia showed higher $\mathrm{NaCl}$ tolerance than the other tested fungal genera. There are a few studies of salt tolerance and mechanisms in marine fungi for example; marine isolates of Trichoderma atroviride and T. asperelloides were reported tolerate $\mathrm{NaCl}$ at $3 \%{ }^{33}$. The thick cell wall and large numbers of vacuoles in marine fungal cells may help these fungi adapt to marine environments ${ }^{48}$ and the increase of the multifunctional cell-wall proteins hydrophobins may played a key role in salt tolerance in eukaryotes ${ }^{49}$. Although the tested marine-derived fungi could grow on media amended with $\mathrm{NaCl}$, the effects of $\mathrm{NaCl}$ on fungal growth and their sporulation were observed in all except E. chevalieri (KUFA0464), which is not surprising because the genus Eurotium is a well-known halophilic and/or xerophilic fungi which is often found in salty food and hypersaline areas ${ }^{50,51}$. These observations corresponded to a previous report which found that $\mathrm{NaCl}$ caused abnormal conidiophore production in Aspergillus species ${ }^{52}$.

Climatic changes such as higher temperatures and drought will result in increased soil salinity, which is predicted to affect plant pathogen growth, development and survival rates as well as modify their pathogenicity leading to changes in disease severity on crops ${ }^{53-54}$. Hence, new BCAs with halo tolerant properties should be urgently sought. In this effort, the results from this preliminary study showed that marinederived fungi are the promising sources of BCAs for application in crop production in normal and salty soil areas and in arid-zone agriculture as well as in supporting crop production under climatic changes in the future.

Results from this study indicate that some of the marine-derived fungi tested in this study possess antagonistic mechanisms including competition for space and nutrients as well as antibiosis production resulting in inhibition the mycelial growth of plant pathogenic fungi. They also possess halo tolerance which made it possible for them to grow on media amended with $7 \% \mathrm{NaCl}$.
These data suggested that they are potential BCAs which may be promising alternatives to the use of synthetic fungicides to control plant diseases in normal and salty soil areas and in arid-zone agriculture. However, further studies are needed to identify antifungal substances responsible in inhibiting mycelial growth of plant pathogenic fungi as well as to evaluate their biocontrol potential against plant disease under greenhouse and field conditions.

\section{ACKNOWLEDGEMENTS}

The authors wish to thank the Graduate School, Kasetsart University and the Kasetsart University Research Development Institute (KURDI) for financial support of this project.

\section{CONFLICT OF INTEREST}

The authors declare that there is no conflicts of interest.

\section{REFERENCES}

1. Naraghi L, Heydari A, Rezaee S, Razavi M. Biocontrol agent Talaromyces flavus stimulates the growth of cotton and potato. J Plant Growth Regul. 2012; 31:471-77.

2. Bjelajac Z, Mijatovic MD, Kozar V. Review of the uncontrolled use of certain chemicals and their adverse effect on human health and safe environment. Oxid Commun. 2015; 38:722-33.

3. Xu X-M, Jeffries P, Pautasso $M$, Jeger MJ. Combined use of biocontrol agents to manage plant diseases in theory and practice. Phytopathology. 2011; 101:102431.

4. Sarrocco S, Diquattro S, Avolio F, Cimmino A, Puntoni G, Doveri F, Evidente A, Vannacci G. Bioactive metabolites from new or rare fimicolous fungi with antifungal activity against plant pathogenic fungi. Eur J Plant Pathol. 2015; 142:61-71.

5. Mehbub MF, Lei J, Franco C, Zhang W. Marine sponge derived natural products between 2001 and 2010: trends and opportunities for discovery of bioactives. Mar Drugs. 2014; 12:4539-77.

6. Hong J-H, Jang S, Heo YM, Min M, Lee $\mathrm{H}$, Lee YM, Lee $\mathrm{H}$, Kim J-J. Investigation of marine-derived fungal diversity and their exploitable biological activities. Mar Drugs. 2015; 13:4137-55.

7. Kathiresan K, Balagurunathan R, Selvam MM. Fungicidal activity of marine actinomycetes against phytopathogenic fungi. Indian J Biotechnol. 2005; 4:271-76.

8. May Zin WW, Prompanya C, Buttachon S, Kijjoa A. Bioactive secondary metabolites from a Thai collection of soil and marine-derived fungi of the genera Neosartorya and Aspergillus. Curr Drug Deliv. 2016; 13:378-88.

9. Devarajan PT, Suryanarayanan TS, Geetha V. 
Endophytic fungi associated with the tropical seagrass Halophila ovalis (Hydrocharitaceae). Indian Journal of Geo-Marine Sciences. 2002; 31:73-4.

10. Dethoup T, Kumla D, Kijjoa A. Mycocidal activity of crude extracts of marine-derived beneficial fungi against plant pathogenic fungi. J Biopest. 2015; 7:107-15.

11. Dethoup T, Gomes NGM, Chaopongpang S, Kijjoa A. Aspergillus similanensis sp. nov. from a marine sponge in Thailand. Mycotaxon. 2016; 131:7-15.

12. Gomes NGM, Bessa LJ, Buttachon S, Costa PM, Buaruang J, Dethoup T, Silva AMS, Kijjoa A. Antibacterial and antibiofilm activity of tryptoquivalines and meroditerpenes from marine-derived fungi Neosartorya paulistensis, N. laciniosa, N. tsunodae, and the soil fungi $N$. fischeri and $N$. siamensis. Mar Drugs. 2014; 12:822-39.

13. Kumla D, Dethoup $T$, Buttachon $S$, Singburaudom N, Silva AMS, Kijjoa A. Spiculisporic acid E, a new spiculisporic acid derivatives from the marine-sponge associated fungus Talaromyces trachyspermus (KUFA 0021). Nat Prod Commun. 2014; 9:1147-50.

14. Buttachon S, May Zin WW, Dethoup T, Gales L, Pereira JA, Silva AMS, Kijjoa A. Secondary metabolites from the culture of the marine sponge-associated fungi Talaromyces tratensis and Sporidesmium circinophorum. Planta Med. 2016; 82:888-96.

15. Noinart J, Buttachon S, Dethoup T, Gales L, Pereira JA, Urbatzka R, Freitas $S$, Lee $M$, Silva AMS, Pinto MMM, Vasconcelos V, Kijjoa A. A new ergosterol analog, a new bis-anthraquinone and anti-obesity activity of anthraquinones from the marine-sponge-associated fungus Talaromyces stipitatus KUFA 0207. Mar Drugs. 2017; 15:139.

16. Höller U, Wright AD, Matthée GF, König GM, Draeger $\mathrm{S}$, Aust $\mathrm{H}-\mathrm{J}$, Schulz B. Fungi from marine sponges: diversity, biological activity and secondary metabolites. Mycol Res. 2000; 104:1354-65.

17. Bhadury P, Mohammad BT, Wright PC. The current status of natural products from marine fungi and their potential as anti-infective agents. J Ind Microbiol Biotechnol. 2006; 33:325-37.

18. Paz Z, Komon-Zelazowska M, Druzhinina IS, Aveskamp MM, Shnaiderman A, Aluma Y, Carmeli S, Ilan M, Yarden O. Diversity and potential antifungal properties of fungi associated with a Mediterranean sponge. Fungal Divers. 2010; 42:17-26.

19. Bugni TS, Ireland CM. Marine-derived fungi: a chemically and biologically diverse group of microorganisms. Nat Prod Rep. 2004; 21:143-63.

20. Debbab A, Aly AH, Liu WH, Proksch P. Bioactive compounds from marine bacteria and fungi. Microb Biotechnol. 2010; 3:544-63.

21. Greve H, Mohamed IE, Pontius A, Kehraus S, Gross H, König GM. Fungal metabolites: structural diversity as incentive for anticancer drug development. Phytochem Rev. 2010; 9:537-45.

22. Thomas TRA, Kavlekar DP, LokaBharathi PA. Marine drugs from sponge-microbe association. Mar Drugs. 2010; 8:1417-68.

23. Debbab A, Aly AH, Proksch P. Endophytes and associated marine derived fungi-ecological and chemical perspectives. Fungal Divers. 2012; 5:45-83.

24. Gao Z, Li B, Zheng C, Wang G. Molecular detection of fungal communities in the Hawaiian marine sponges Suberites zeteki and Mycale armata. Appl Environ Microbiol. 2008; 74:6091-101.

25. Wang G, Li Q, Zhu P. Phylogenetic diversity of cultivable fungi associated with the Hawaiian sponge Suberites zeteki and Gelliodes fibrosa. Antonie van Leeuwenhoek. 2008; 3:163-74.

26. Li Z. Advances in marine microbial symbionts in the China Sea and related pharmaceutical metabolites. Mar Drugs. 2009; 7:113-29.

27. Li Q, Wang $G$. Diversity of fungal isolates from three Hawaiian marine sponges. Microbiol Res. 2009; 164:233-41.

28. Ding B, Yin Y, Zhang F, Li Z. Recovery and phylogenetic diversity of culturable fungi associated with marine sponges Clathrina luteoculcitella and Holoxea sp. in the South China Sea. Mar Biotechnol. 2011; 13:713-21.

29. Kohlmeyer J, Kohlmeyer E. 1979. Marine mycology: the higher fungi. Academic Press, London, England.

30. Rongbian WEI, Fuchao LI, Song R, Song QIN. Comparison of two marine sponge-associated Penicillium strains DQ25 and SC10: differences in secondary metabolites and their bioactivities. Ann Microbiol. 2009; 59:579-85.

31. Manilal A, Sabarathnam B, Kiran GS, Sujith S, Shakir $C$, Selvin J. Antagonistic potentials of marine sponge associated fungi Aspergillus clavatus MFD15. Asian Journal of Medical Sciences. 2010; 2:195-200.

32. Shen S, Li W, Wang J. Antimicrobial and antitumor activities of crude secondary metabolites from a marine fungus Penicillium oxalicum 0312F. Afr $J$ Microbiol Res. 2014; 8:1480-85.

33. Gal-Hemed I, Atanasova L, Komon-Zelazowska M, Druzhinina IS, Viterbo A, Yarden O. Marine isolates of Trichoderma spp. as potential halotolerant agents of biological control for arid-zone agriculture. Appl Environ Microbiol. 2011; 77:5100-09.

34. Murray MG, Thompson WF. Rapid isolation of high molecular weight plant DNA. Nucleic Acids Res. 1980; 8:4321-25.

35. White TJ, Bruns T, Lee S, Taylor J. 1990. Amplification and direct sequencing of fungal ribosomal RNA genes for phylogenetics. In: Innis MA, Gelfand DH, Sninsky JJ, White TJ (eds.). PCR protocols: a guide to methods and applications. Academic Press, New York, USA.

36. Cai F, Yu G, Wang P, Wei Z, Fu L, Shen Q, Chen W. Harzianolide, a novel plant growth regulator and systemic resistance elicitor from Trichoderma harzianum. Plant Physiol Biochem. 2013; 73:106-13.

37. Bae S-J, Mohanta TK, Chung JY, Ryu M, Park G, Shim S, Hong S-B, Seo H, Bae D-W, Bae I, Kim J-J, Bae H. Trichoderma metabolites as biological control agents against Phytophthora pathogens. Biol Control. 2016; 92:128-38.

38. El-Kassas HY, Khairy HM. A trial for biological control of a pathogenic fungus (Fusarium solani) by some marine microorganisms. American-Eurasian J Agric \& Environ Sci. 2009; 5:434-40.

39. Devi P, Wahidulla S, Kamat T, D'Souza L. Screening marine organisms for antimicrobial activity against 
clinical pathogens. Indian Journal of Geo-Marine Sciences. 2011; 40:338-46.

40. Lozano-Tovar MD, Ortiz-Urquiza A, Garrido-Jurado I, Trapero-Casas A, Quesada-Moraga E. Assessment of entomopathogenic fungi and their extracts against a soil-dwelling pest and soil-borne pathogens of olive. Biol Control. 2013; 67:409-20.

41. Xie LW, Jiang SM, Zhu HH, Sun W, Ouyang YC, Dai SK, Li $X$. Potential inhibitors against Sclerotinia sclerotiorum, produced by the fungus Myrothecium sp. associated with the marine sponge Axinella sp. Eur J Plant Pathol. 2008; 122:571-78.

42. Talubnak C, Soythong K. Biological control of vanilla anthracnose using Emericella nidulans. J Agri Technol. 2012; 6:47-55.

43. Sibounnavong $\mathrm{P}$, Chareonporn $\mathrm{C}$, Kanokmedhakul $\mathrm{S}$, Soytong K. Antifungal metabolites from antagonistic fungi used to control tomato wilt fungus Fusarium oxysporum f.sp. lycopersici. Afr J Biotechnol. 2011; 10:19714-722.

44. Kumsorn W. 2013. Diversity of Emericella species from soil and in vitro efficacy against plant pathogenic fungi. M.Sc. thesis, Faculty of Agriculture, Kasetsart University. Bangkok, Thailand.

45. Vasanthabharathi V, Jayalakshmi S. Bioactive potential of symbiotic bacteria and fungi from marine sponges. Afr J Biotechnol. 2012; 11:7500-11.

46. Sun SL, Kim MY, Van K, Lee Y-H, Zhong C, Zhu ZD, Lestari
P, Lee Y-W, Lee S-H. First report of Neocosmospora vasinfecta var. vasinfecta causing soybean stem rot in South Korea. Plant Dis. 2014; 98:1744.

47. Greer AM, Spurlock TN, Coker CM. First report of Neocosmospora stem rot of soybean caused by Neocosmospora vasinfecta in Arkansas. Plant Dis. 2015; 99:554.

48. Clipson N, Hooley P. Salt tolerance strategies in marine fungi. Mycologist. 1995; 9:3-5.

49. Plemenitaš $A$, Lenassi $M$, Konte $T$, Kejžar $A$, Zajc $J$, Gostinčar C, Gunde-Cimerman N. Adaptation to high salt concentrations in halotolerant/ halophilic fungi: a molecular perspective. Front Microbiol. 2014; 5:199.

50. Butinar L, Zalar P, Frisvad JC, Gunde-Cimerman N. The genus Eurotium-members of indigenous fungal community in hypersaline waters of salterns. FEMS Microbiol Ecol. 2005; 51:155-66.

51. Hubka V, Kolarík M, Kubátová A, Peterson SW. Taxonomic revision of Eurotium and transfer of species to Aspergillus. Mycologia. 2013; 105:912-37.

52. Tresner HD, Hayes JA. Sodium chloride tolerance of terrestrial fungi. Appl Microbiol. 1971; 22:210-13.

53. Chakraborty S, Tiedemann AV, Teng PS. Climate change: potential impact on plant diseases. Environ Pollut. 2000; 108:317-26.

54. Elad Y, Pertot I. Climate change impacts on plant pathogens and plant diseases. J Crop Improv. 2014; 28:99-139. 